\title{
Composite Right- and Left-Handed Traveling-Wave Field-Effect Transistors
}

\author{
Koichi Narahara \\ Graduate School of Science and Engineering, Yamagata University, 4-3-16 Jonan, Yonezawa, Yamagata 992-8510, Japan \\ Correspondence should be addressed to Koichi Narahara, narahara@yz.yamagata-u.ac.jp \\ Received 27 March 2012; Revised 27 June 2012; Accepted 11 July 2012 \\ Academic Editor: Egidio Ragonese \\ Copyright () 2012 Koichi Narahara. This is an open access article distributed under the Creative Commons Attribution License, \\ which permits unrestricted use, distribution, and reproduction in any medium, provided the original work is properly cited. \\ We introduce a composite right- and left-handed travelling-wave field-effect transistor (CRLH TWFET) for developing large- \\ scale platform to support left-handed waves. The device represents two electromagnetically coupled CRLH transmission lines by \\ capacitance and FET transconductance. Owing to the couplings, two different modes can support waves in CRLH TWFETs. It was \\ experimentally established that waves supported by one of the modes were amplified, while those supported by the other mode \\ were significantly attenuated. To quantify the wave propagation in CRLH TWFETs, we developed a numerical model based on \\ the transmission line theory that well simulated measured results. This paper discusses the results of numerical calculations that \\ validate the design criteria of CRLH TWFETs.
}

\section{Introduction}

Composite right- and left-handed (CRLH) transmission lines (TLs) have been extensively studied and several important breakthroughs have been achieved in the management of electromagnetic continuous waves [1]. CRLH TLs have a single series and shunt inductance-capacitance (LC) pair in each unit cell. They exhibit lefthandedness; that is, at certain frequencies, their phase velocity has a sign opposite to that of their group velocity. Figure 1 shows the unit cell of a CRLH TL, where $L_{R}, C_{L}, L_{L}$, and $C_{R}$ are the series inductance, series capacitance, shunt inductance, and shunt capacitance, respectively. The series LC pair consists of $L_{R}$ and $C_{L}$, and the shunt pair consists of $L_{L}$ and $C_{R}$. These parameters define the upper and lower zero-wavelength frequencies of the CRLH TL, which are given by $\omega_{u}=\max \left(1 /\left(C_{L} L_{R}\right)^{1 / 2}, 1 /\left(C_{R} L_{L}\right)^{1 / 2}\right)$ and $\omega_{l}=\min \left(1 /\left(C_{L} L_{R}\right)^{1 / 2}, 1 /\left(C_{R} L_{L}\right)^{1 / 2}\right)$, respectively. The line exhibits righthandedness at frequencies greater than $\omega_{u}$ and left-handedness at frequencies lower than $\omega_{l}$. A frequency band gap exists between $\omega_{l}$ and $\omega_{u}$ in which all supporting modes are evanescent.

The use of CRLH TLs may sometimes be impractical because of their finite electrode resistance and substrate current leakage, and may require loss compensation schemes. Recently, several authors have investigated methods for amplifying LH waves in 1D using transistors [2-4].
In particular, we analyse the use of two CRLH TLs that interact continuously via transistor transconductance together with both capacitive and inductive couplings, which we call a CRLH travelling-wave field-effect transistor (TWFET), or CRLH TWFET for brevity.

There are two different schemes to achieve loss compensation or amplification of left-handed waves. One of the methods is to interconnect passive CRLH sections with amplifier section. Based on this methodology, CasaresMiranda et al. [2] fabricated a leaky-wave antenna, whose gain was much enhanced compared to its passive counterpart. Multiple cascades of the interconnecting sections enable extension of the effective propagation length of a LH wave. However, a phase jump occurs at the amplifier section. Another method is continuous amplification. Si et al. [3] proposed a microwave CRLH TL incorporating ideal gain devices in each unit cell. They demonstrated a simple circuit cell consisting of two FETs operated as such gain devices. On the other hand, Maezawa et al. [4] proposed a CRLH TL periodically loaded with resonant tunnel diode (RTD) pairs. The negative differential resistance (NDR) of RTDs can compensate the loss in passive CRLH TLs. It is suggested that the line can amplify signals traveling along the line by the NDR of the RTD. In either case, the unit-cell structure is much simpler than CRLH TWFETs. 


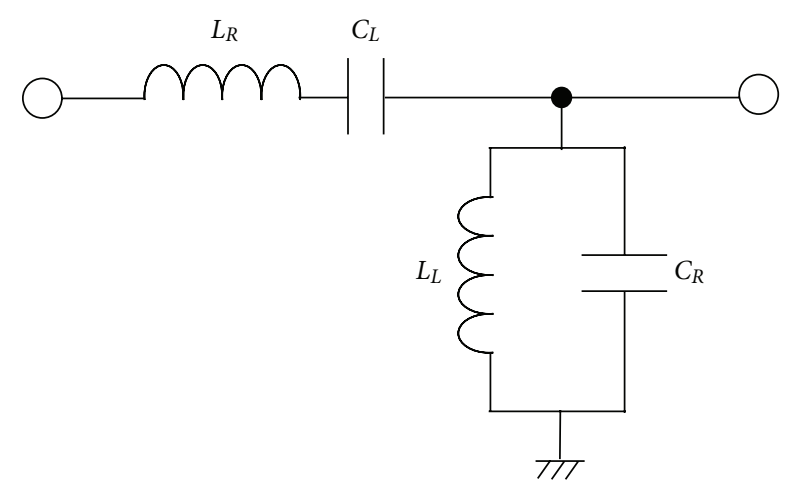

Figure 1: Unit cell of CRLH TL.

The CRLH TL has inductance-capacitance pairs in each unit cell. With the simple introduction of an NDR device, these pairs can exhibit resonant behaviour. We carried out the stability analysis with respect to CRLH TWFETs to establish the fact that they succeed in suppressing instability and amplifying the LH waves [5]. On the other hand, as far as we know, such stability analysis has not been carried out for the schemes that employ NDR device in each unit cell. Table 1 summarizes the merits and demerits of the above-mentioned three platforms for compensating attenuation of $\mathrm{LH}$ waves.

In the following, we first clarify our design concept of CRLH TWFETs. We then discuss several fundamental properties of a CRLH TWFET including the device configuration, dispersion relationship, and design criteria for wave amplification and termination schemes. Next, we describe the numerical model to simulate the measured results. Then, we numerically confirm the validity of the design criteria of a CRLH TWFET.

\section{Design Concept of CRLH TWFETs}

Figure 2 illustrates our design concept of CRLH TWFETs. Owing to couplings, two different propagation modes, called the $\mathrm{c}$ - and $\pi$-modes, can support waves in CRLH TWFETs. Each mode has its own speed, characteristic impedance, and the voltage fraction between the gate and drain lines. In general, the c-mode waves have the even parity between the gate and drain lines. On the other hand, the $\pi$-mode waves have the odd parity between the gate and drain lines.

Our design concept stems from the fact that a CRLH TWFET can amplify the waves carried by one of two modes, and attenuate ones carried by the other mode significantly. We can exclude the influences caused by waves carried by the attenuating mode to suppress modal dispersions. Moreover, wave reflection at the ends can be minimized by the matched terminations with the characteristic impedances corresponding to the amplifying mode.

At present, we consider the case where the $\pi$-mode is the unique one to be amplified. Then, the terminal resistances $R_{\mathrm{Tg}}$ and $R_{\mathrm{Td}}$ are set equal to the $\pi$-mode characteristic impedances for the gate and drain lines, respectively. In order to develop the waves carried by the $\pi$-mode, the input ports
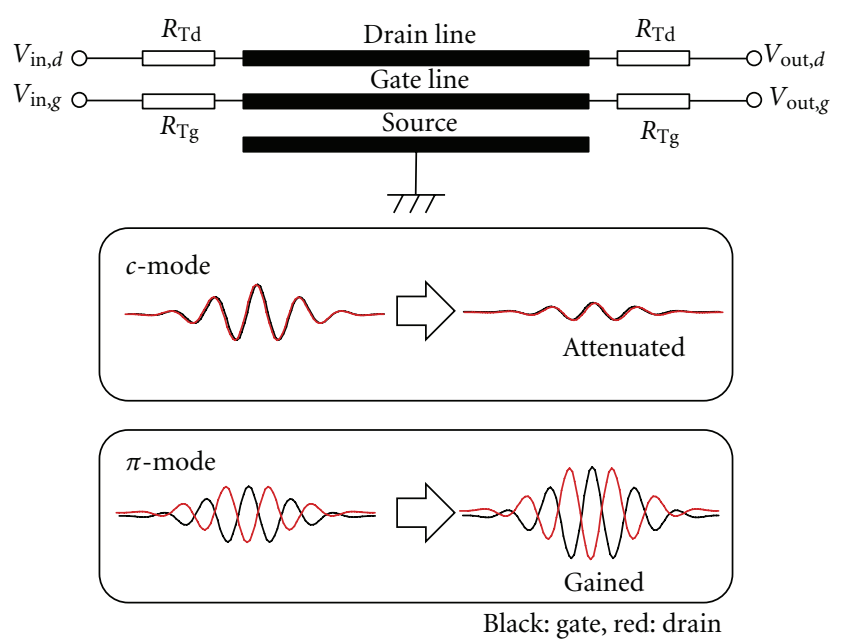

FIgUre 2: Design concept of CRLH TWFETs.

$V_{\mathrm{in}, g}$ and $V_{\mathrm{in}, d}$ are excited by the waves satisfying the $\pi$ mode voltage fraction. In this study, we describe the results of numerical calculations using a model of a CRLH TWFET based on the elemental transmission line theory. The model well simulates the measured properties of the device and can evaluate the contributions of the finite series inductors to both LH and RH waves travelling in a line. The operation bandwidth is limited by the FET cutoff frequency. Moreover, the influences of parasitic inductance and capacitance, which cannot be assumed a priori, increase at high frequencies. However, as far as the line is based on the print-circuit-board (PCB) technologies, we can well suppress the influences of parasitic elements, whose operation bandwidth presently extends the microwave frequencies.

\section{CRLH TWFETS}

Figure 3 shows the circuit configuration of the unit cell of a CRLH TWFET. Each unit cell includes an FET. For the gate (drain) line, $L_{R g(d)}, C_{L g(d)}, L_{L g(d)}$, and $C_{R g(d)}$ represent the series inductor, series capacitor, shunt inductor, and shunt capacitor, respectively. The gate line is connected with the gate electrode of the FET, whose drain electrode is connected with the drain line. The source electrode is grounded. Note that the biasing voltages to the gate $\left(V_{G}\right)$ and drain $\left(V_{D}\right)$ of the FET can be rendered via $L_{L g}$ and $L_{L d}$, respectively. Every inductor accompanies finite parasitic resistance, which is denoted as $R_{G}, R_{D}, R_{\mathrm{in}, g}$, and $R_{\mathrm{in}, d}$ for $L_{R g}, L_{R d}, L_{L g}$, and $L_{L d}$, respectively. The drain-source current of an FET is modelled as $I_{d s}\left(V_{G}\right)=\beta\left(V_{G}-V_{\text {th }}\right)^{2}$, where $\beta, V_{t h}$, and $V$ are the transconductance coefficient, threshold voltage, and the gate line voltage, respectively. For analytical evaluations, we consider the small-signal counterpart of $I_{d s}$, that is, $I_{d s}\left(V_{G}\right)=$ $G_{m}\left(V_{G}-V_{\text {th }}\right)$, where $G_{m}$ is the transconductance given by $2 \beta\left(V_{G}-V_{\text {th }}\right)$. Moreover, an FET includes the gate-source capacitance $C_{g s}$, gate-drain capacitance $C_{g d}$, and drainsource capacitance $C_{d s}$. These FET parasitic capacitances can be embedded in elements in Figure 2. Concretely, $C_{g s}, C_{d s}$, and $C_{g d}$ can be included in $C_{R g}, C_{R d}$, and $C_{m}$, respectively. 
TABLE 1: Figure of merits of amplifying LH waves.

\begin{tabular}{lccc}
\hline & Cascades of CRLH TL and amplifier sections & Unit cell with NDR devices/circuits & CRLH TWFET \\
\hline Design flexibility & + & + & - \\
Phase continuity & - & + & + \\
Verification & + & + & + \\
Stability & + & \pm & + \\
\hline
\end{tabular}

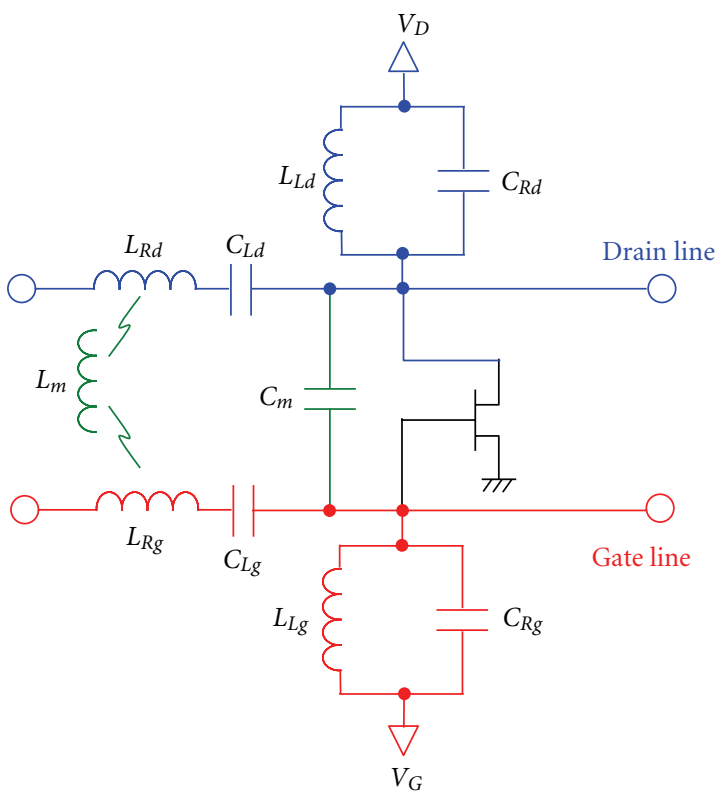

FIgURE 3: Unit cell of CRLH TWFET. Both the gate and drain lines have the CRLH TL structures. These two lines are coupled via the mutual inductance $L_{m}$, mutual capacitance $C_{m}$, and FET contributions.

Owing to the presence of the passive coupling between the gate and drain lines, two different propagation modes can carry the waves [6].

Each mode has its own dispersion, characteristic impedance, and the voltage fraction between the lines. Figure 4 shows a typical dispersion relationship of each mode. To obtain this, we set $L_{R g}, C_{L g}, L_{L g}$, and $C_{R g}$ to $0.75 \mathrm{nH}$, $1.5 \mathrm{pF}, 1.25 \mathrm{nH}$, and $1.0 \mathrm{pF}$, respectively. Moreover, $L_{R d}, C_{L d}$, $L_{L d}, C_{R d}$, and $C_{m}$ are set to $1.0 \mathrm{nH}, 2.0 \mathrm{pF}, 0.5 \mathrm{nH}, 1.5 \mathrm{pF}$, and $0.2 \mathrm{pF}$, respectively. There are mostly two different modes for a given frequency, although only the unique mode is allowed in the filled frequencies in Figure 4. For definiteness, we call the fast and slow modes the $\pi$ and $c$, respectively. Accordingly, the solid and dotted curves correspond to the $c$ - and $\pi$ modes, respectively. The upper (lower) two exhibit RH (LH) properties.

By inputting the voltage signals having the $c-(\pi-)$ mode voltage fraction between the lines, they are carried by the $c$ $(\pi-)$ mode. In addition, the matched termination with the $c^{-}$ $(\pi-)$ mode characteristic impedance suppresses the multiple reflections that terribly distort the waves in the line. When the voltage fraction is positive (negative), the corresponding mode becomes in phase (out of phase).

We consider the cases when a small $G_{m}$ is introduced. We can then obtain the $c^{-}(\pi-)$ mode per-unit-cell gain $\alpha_{c(\pi)}$ by

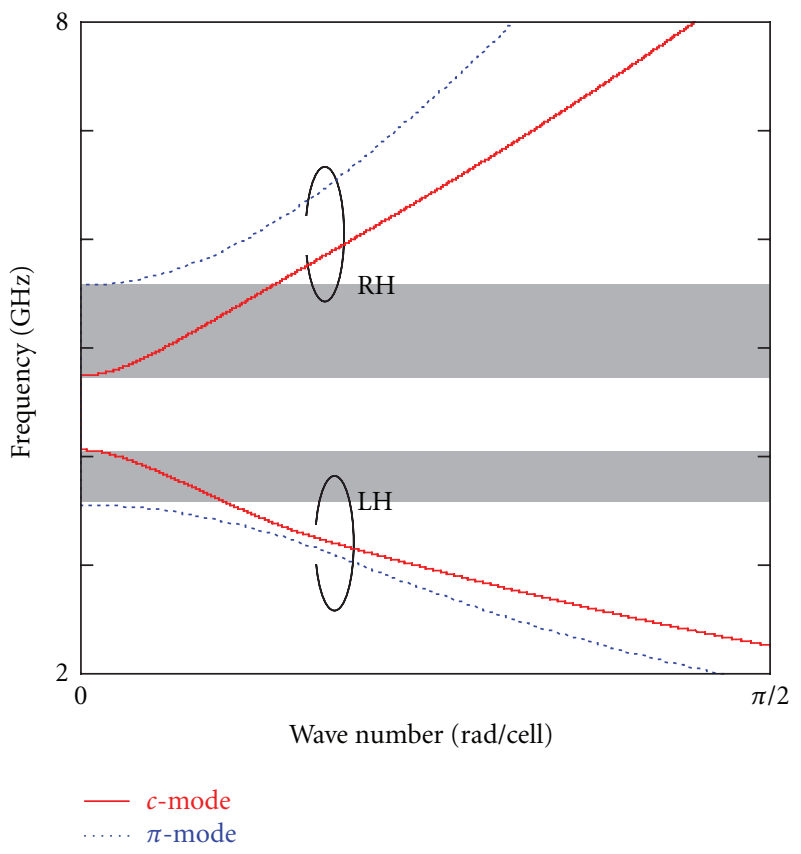

Figure 4: Dispersive property of CRLH TWFET. The filled frequency regions are sandwiched by zero-wavelength frequencies, which are $3.6,4.1,4.7$, and $5.6 \mathrm{GHz}$ in ascending order.

series-expanding the complex dispersion with respect to $G_{m}$, which is given by

$$
\begin{aligned}
& \alpha_{c}=-\frac{v_{c} L_{m} G_{m}}{4\left(1 / v_{c}^{2}-1 / v_{\pi}^{2}\right)}\left(\frac{1}{v_{c}^{2}}-\frac{1}{v_{\pi}^{2}}+\frac{1}{v_{m}^{2}}\right), \\
& \alpha_{\pi}=-\frac{v_{\pi} L_{m} G_{m}}{4\left(1 / v_{c}^{2}-1 / v_{\pi}^{2}\right)}\left(\frac{1}{v_{c}^{2}}-\frac{1}{v_{\pi}^{2}}-\frac{1}{v_{m}^{2}}\right),
\end{aligned}
$$

where $v_{c(\pi)}$ represents the phase velocity of the $c-(\pi-)$ mode. Moreover, $v_{m}$ is defined by

$$
\begin{aligned}
\frac{1}{v_{m}^{2}}= & -\frac{2 C_{m}}{C_{L g} C_{L d} L_{m} \omega^{4}}\left(\frac{\omega^{2}}{\omega_{\text {seg }}^{2}}-1\right)\left(\frac{\omega^{2}}{\omega_{\text {sed }}^{2}}-1\right) \\
& +C_{m}\left(\frac{1}{C_{L g}}\left(\frac{1}{\omega_{\text {seg }}^{2}}-\frac{1}{\omega^{2}}\right)+\frac{1}{C_{L d}}\left(\frac{1}{\omega_{\text {sed }}^{2}}-\frac{1}{\omega^{2}}\right)\right) \\
& +\frac{\omega_{L g}^{2}}{\omega^{4}}\left(1-\frac{\omega^{2}}{\omega_{\text {sed }}^{2}}-\frac{\omega^{2}}{\omega_{\text {shg }}^{2}}\right)+\frac{\omega_{L d}^{2}}{\omega^{4}}\left(1-\frac{\omega^{2}}{\omega_{\text {sed }}^{2}}-\frac{\omega^{2}}{\omega_{\text {shd }}^{2}}\right) \\
& +\frac{1}{\omega_{R g}^{2}}+\frac{1}{\omega_{R d}^{2}},
\end{aligned}
$$




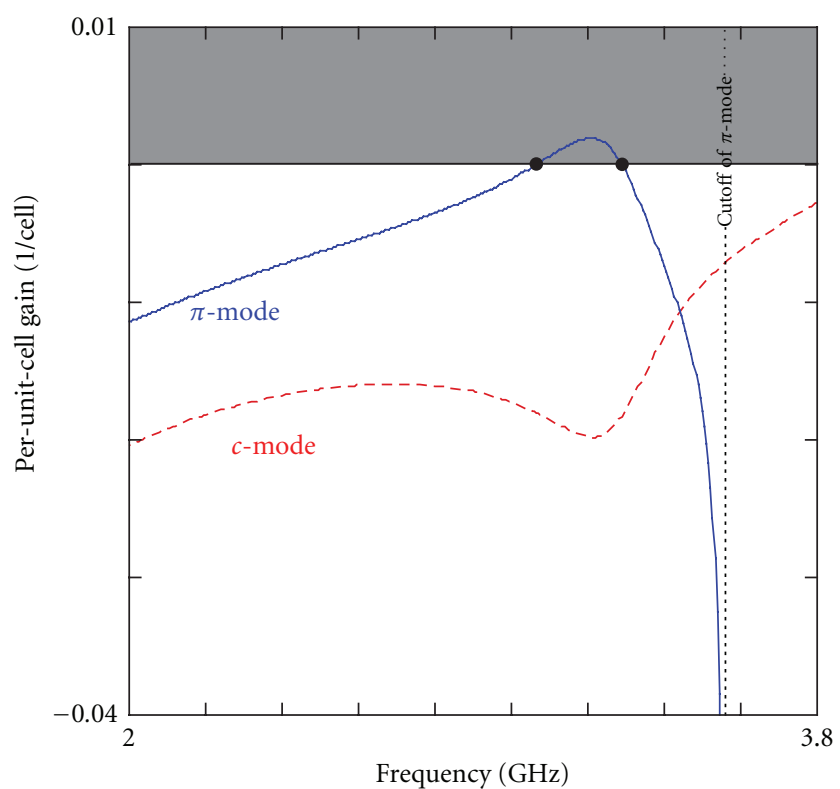

FIGURE 5: Per-unit-cell gain for LH frequencies. The solid and dashed curves represent the $\pi$ - and $c$-modes, respectively. The filled region corresponds to amplification.

where $\omega_{R g}=1 /\left(L_{R g} C_{R g}\right)^{1 / 2}, \omega_{R d}=1 /\left(L_{R d} C_{R d}\right)^{1 / 2}, \omega_{L g}=$ $1 /\left(L_{L g} C_{L g}\right)^{1 / 2}, \omega_{L d}=1 /\left(L_{L d} C_{L d}\right)^{1 / 2}, \omega_{\text {seg }}=1 /\left(L_{R g} C_{L g}\right)^{1 / 2}$, $\omega_{\text {sed }}=1 /\left(L_{R d} C_{L d}\right)^{1 / 2}, \omega_{\text {shg }}=1 /\left(L_{L g} C_{R g}\right)^{1 / 2}$, and $\omega_{\text {shd }}=$ $1 /\left(L_{L d} C_{R d}\right)^{1 / 2}$. Note that $v_{m}$ can become imaginary for frequencies for which the RHS of (3) becomes negative. Moreover, (3) becomes singular for $L_{m}=0$. In such a situation, $\alpha_{c(\pi)}$ should be written as

$$
\begin{aligned}
& \alpha_{c}=-\frac{v_{c} C_{m} G_{m}\left(1-\omega^{2} / \omega_{\text {sed }}^{2}\right)\left(1-\omega^{2} / \omega_{\mathrm{seg}}^{2}\right)}{2 \omega^{4} C_{L g} C_{L d}\left(1 / v_{c}^{2}-1 / v_{\pi}^{2}\right)}, \\
& \alpha_{\pi}=-\frac{v_{\pi} C_{m} G_{m}\left(1-\omega^{2} / \omega_{\mathrm{sed}}^{2}\right)\left(1-\omega^{2} / \omega_{\mathrm{seg}}^{2}\right)}{2 \omega^{4} C_{L g} C_{L d}\left(1 / v_{c}^{2}-1 / v_{\pi}^{2}\right)} .
\end{aligned}
$$

In the above, $\alpha_{c(\pi)}$ is defined to be negative for amplifying $\mathrm{RH}$ waves. Correspondingly, the amplitude of the wave increases if $\alpha_{c(\pi)}$ becomes positive for $\mathrm{LH}$ waves, because the phase velocity has the opposite sign from the group velocity. By definitions, $v_{\pi}$ is always greater than $v_{c}$; therefore, $\alpha_{c} \alpha_{\pi}<$ 0 for any frequencies if $L_{m}$ is negligible. It is concluded that one of the two modes can be uniquely amplified. More specifically, the $c-(\pi-)$ mode can be uniquely amplified for LH $(\mathrm{RH})$ waves if $\omega<\omega_{\text {sel }}$ or $\omega>\omega_{\text {seu }}$, where $\omega_{\text {seu }}=$ $\max \left(\omega_{\text {seg }}, \omega_{\text {sed }}\right)$ and $\omega_{\text {sel }}=\min \left(\omega_{\text {seg }}, \omega_{\text {sed }}\right)$. On the other hand, the $c^{-}(\pi-)$ mode can be uniquely amplified for RH (LH) waves if $\omega_{\text {sel }}<\omega<\omega_{\text {seu }}$.

By the introduction of any small but finite resistance $R$, we obtain the form $\alpha_{c, \pi}=p_{c, \pi} G_{m}+q_{c, \pi} R$ with some coefficients $p_{c, \pi}$ and $q_{c, \pi}$ for complex dispersion. Irrespective of $q_{c, \pi}$, only one of the two modes is possibly amplified, if $p_{c} p_{\pi}<0$. Thus, the key criteria that a CRLH TWFET can be designed to satisfy the condition for the unique mode to be amplified are equally valuable even with any loss or leakage.

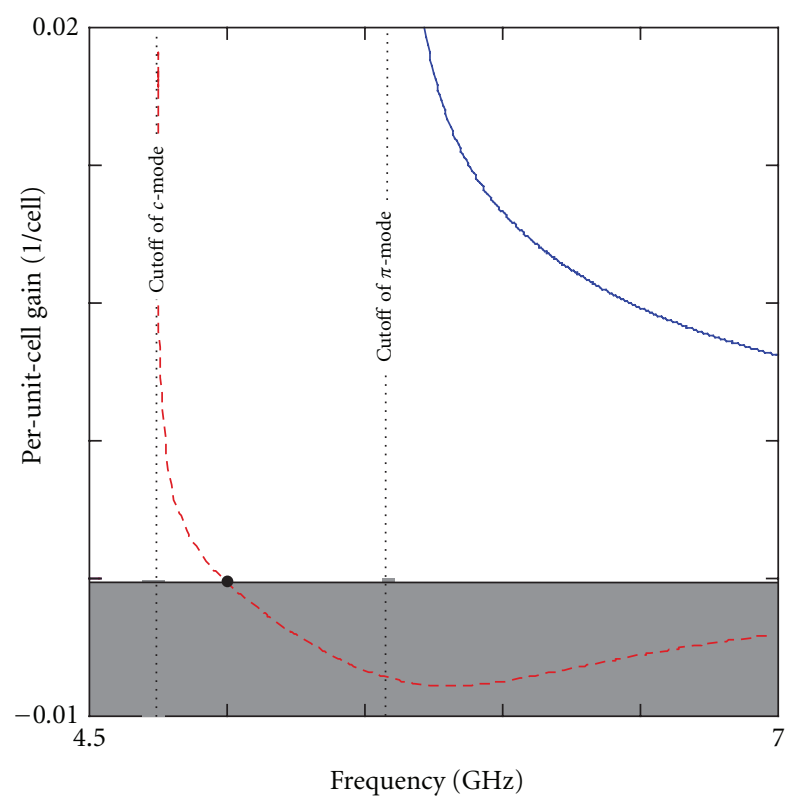

FIgURE 6: Per-unit-cell gain for RH frequencies. The solid and dashed curves represent the $\pi$ - and $c$-modes, respectively. The filled region corresponds to amplification.

In general, the resistance becomes larger for higher frequencies. It tends to reach a saturated value. Instead of modelling this frequency dependence, we employ the saturated resistance for all the frequencies for overestimating. We assigned the values of parasitic resistance to be proportional to the inductance, such that the resistance for $1.0-\mathrm{nH}$ inductor becomes $0.15 \Omega$. By adding the linearized contribution of resistances in per-unit-cell gain, we obtain Figures 5 and 6 , setting $G_{m}$ to $2.0 \mathrm{mS}$. Figure 5 shows the per-unit-cell gain for low frequencies corresponding to the LH branches. The amplified waves are expected only for the $\pi$-mode at the frequencies between 3.0 and $3.3 \mathrm{GHz}$. The gain becomes maximal at $3.2 \mathrm{GHz}$, where the discrepancy between $v_{c}$ and $v_{\pi}$ becomes minimal. Figure 6 is for high frequencies corresponding to the $\mathrm{RH}$ branches. The amplified waves are expected only for the $c$-mode at the frequencies greater than $4.95 \mathrm{GHz}$.

\section{Numerical Simulation of Test Line}

Recently, we experimentally characterized a CRLH TWFET using discrete components on a breadboard [7]. We built a 90 -section device. For convenience, all the series inductances were eliminated. As a result, the test line exhibited LH property for all the frequencies allowed to travel. The transistors used were Toshiba 2SK30A, whose typical threshold voltage was $-1.8 \mathrm{~V}$. The inductors and capacitors were implemented using the TDK SPT0406 series and the TDK FK series, respectively. The values of $C_{L g}, C_{R g}, C_{L d}, C_{R d}$, and $C_{m}$ were set to $470,47,470,470$, and $47 \mathrm{pF}$, respectively. Moreover, the values of $L_{L g}$ and $L_{L d}$ were set to 10.0 and $4.7 \mu \mathrm{H}$, respectively. The gate and drain lines were fed by the envelope pulses generated by an NF WF1974 two-channel function generator. The waveforms were detected by Agilent 


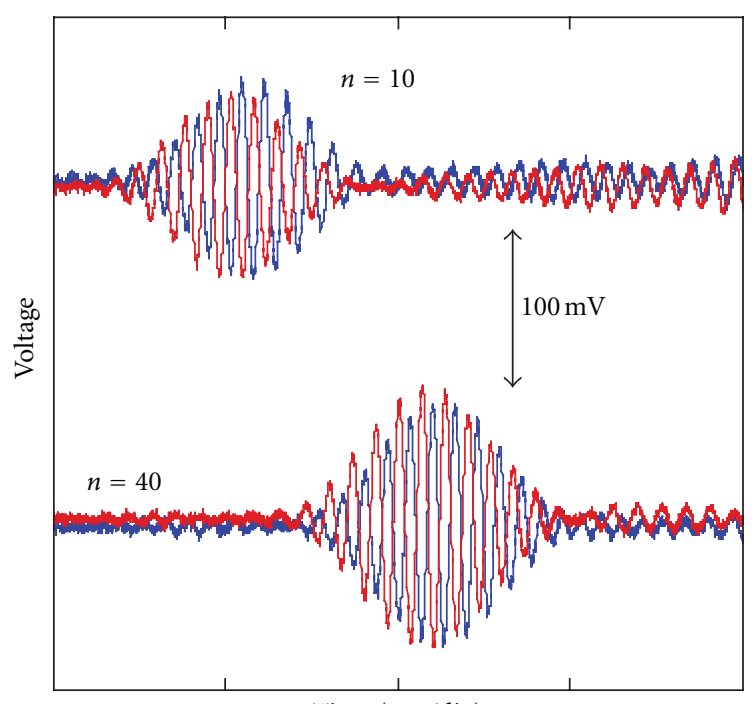

Time $(3 \mu \mathrm{s} / \mathrm{div})$

(a)

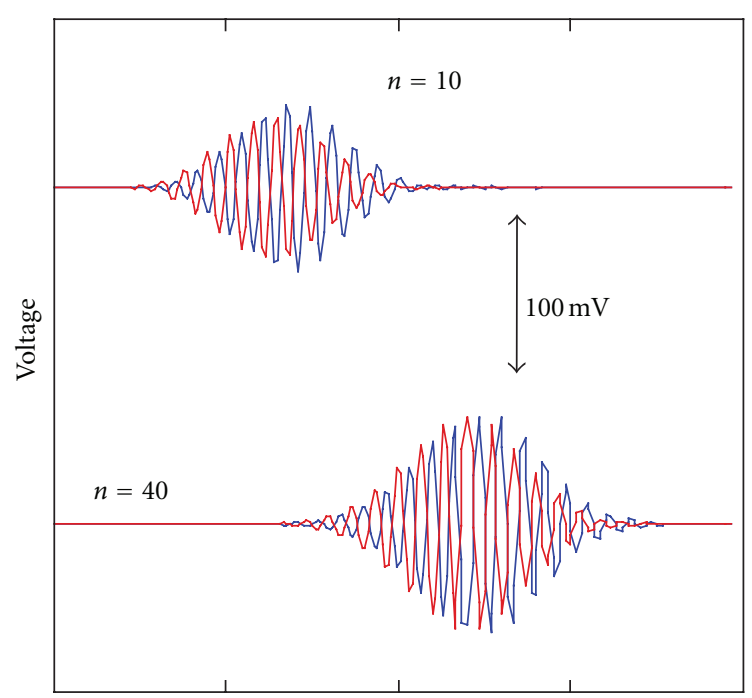

Time $(3 \mu \mathrm{s} / \mathrm{div})$

(b)

Figure 7: Time-domain waveforms in test CRLH TWFET. (a) Measured and (b) calculated ones for the $\pi$-mode envelope pulses.

10073C passive probes and monitored in the time domain by using an Agilent DSO90254A oscilloscope. Through the measurements, we found that the $\pi$-mode gains amplitudes, while the $c$-mode loses them. Moreover, when the test line is terminated with the $\pi$-mode characteristic impedance, the multiple reflections of waves could be effectively suppressed.

To reinforce these observations, we simulate the measured results by solving the transmission equations of the test line using finite-difference time-domain method. The equations are given by

$$
\begin{aligned}
L_{R g} & \frac{d^{2} I_{n}}{d t^{2}}+L_{m} \frac{d^{2} J_{n}}{d t^{2}} \\
& =-\frac{I_{n}}{C_{L g}}-R_{g} \frac{d I_{n}}{d t}-\frac{d}{d t}\left(V_{n}-V_{n-1}\right),
\end{aligned}
$$

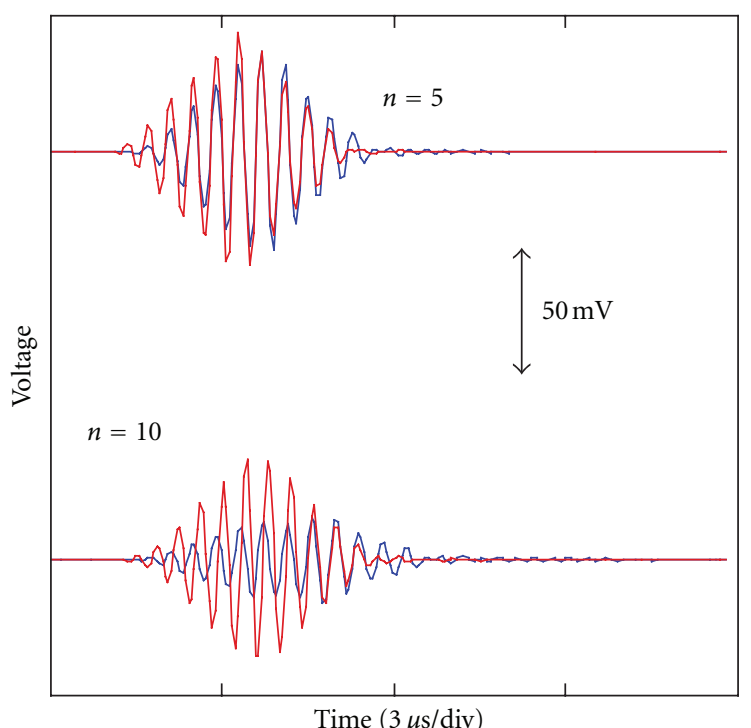

(a)

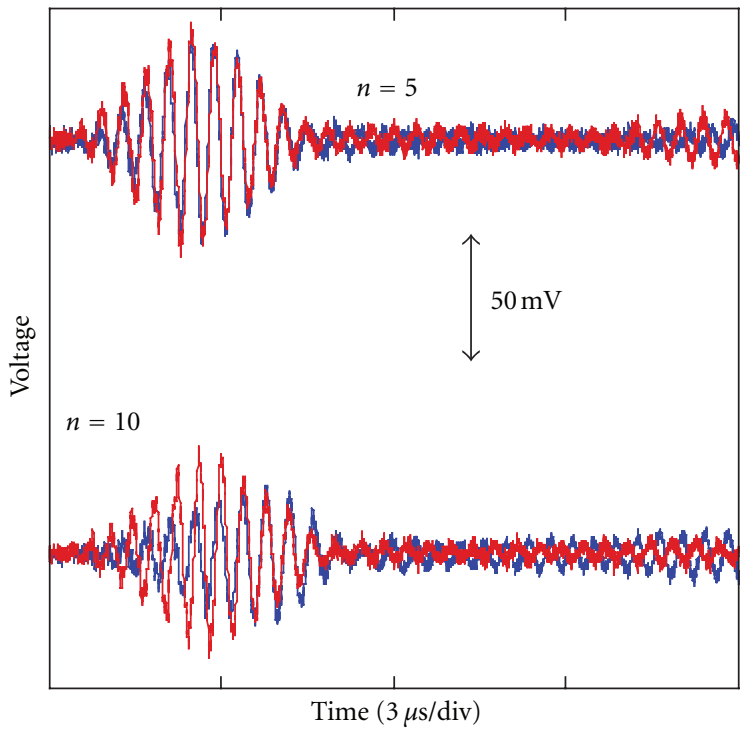

(b)

FIgUre 8: Time-domain waveforms in test CRLH TWFET. (a) Measured and (b) calculated ones for the $c$-mode envelope pulses.

$$
\begin{aligned}
& L_{R d} \frac{d^{2} J_{n}}{d t^{2}}+L_{m} \frac{d^{2} I_{n}}{d t^{2}} \\
& =-\frac{J_{n}}{C_{L d}}-R_{d} \frac{d J_{n}}{d t}-\frac{d}{d t}\left(W_{n}-W_{n-1}\right), \\
& \frac{W_{n}-V_{D}}{L_{L d}} \\
& =\left(\frac{d}{d t}+\frac{R_{\mathrm{in}, d}}{L_{L d}}\right)\left(-\left(C_{R d}+C_{m}\right) \frac{d W_{n}}{d t}\right. \\
& \left.\quad+C_{m} \frac{d V_{n}}{d t}+J_{n}-J_{n+1}-I_{d s}\left(V_{n}\right)\right), \\
& \frac{V_{n}-V_{G}}{L_{L g}}=\left(\frac{d}{d t}+\frac{R_{\mathrm{in}, g}}{L_{L g}}\right) \\
& \quad \times\left(-\left(C_{R g}+C_{m}\right) \frac{d V_{n}}{d t}+I_{n}-I_{n+1} C_{m} \frac{d W_{n}}{d t}\right),
\end{aligned}
$$




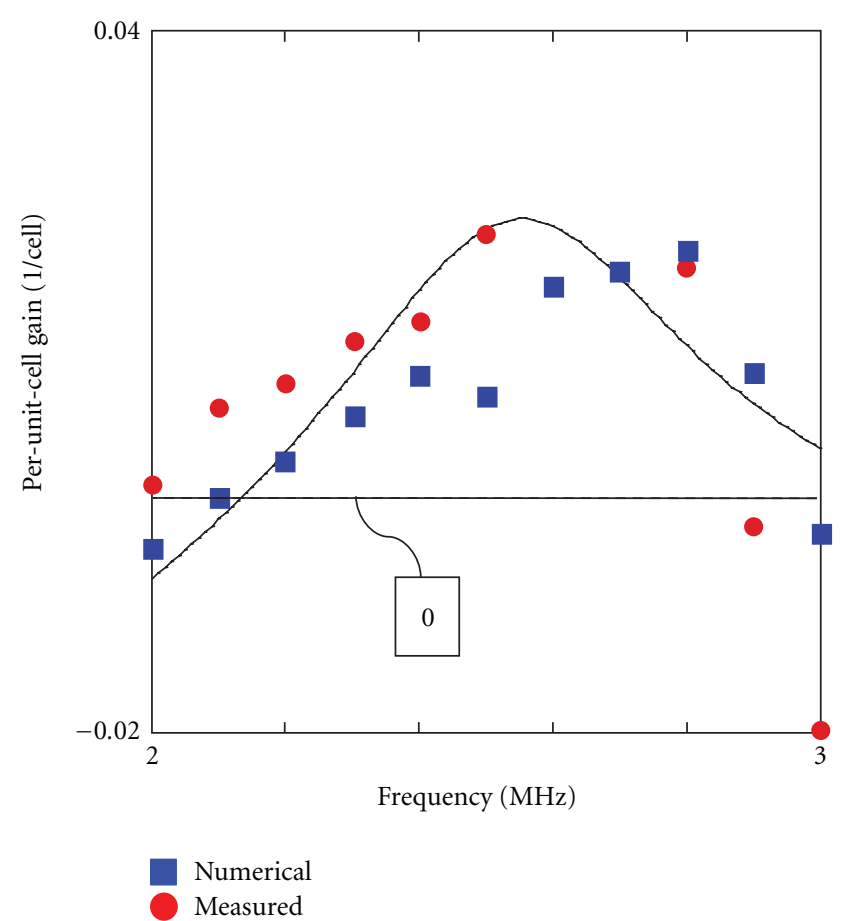

Figure 9: Frequency dependence of per-unit-cell gain. Circles and squares represent the measured and numerical dependences, respectively. Positive gains correspond to wave amplification. The solid curve plots (5) for reference.

where $V_{n}$ and $W_{n}$ represent the gate and drain line voltages at the $n$th cell, respectively. Moreover, $I_{n}$ and $j_{n}$ represent the gate and drain line current flowing into the $n$th cell, respectively. Figures $7(a)$ and $7(b)$ show the measured and calculated waveforms, respectively, when gaussian envelope pulses with a carrier frequency of $2.5 \mathrm{MHz}$ were input at one of the ends with the $\pi$-mode voltage fraction between the gate and drain lines. The upper and lower waveforms were monitored at the 10th and 40th cells, respectively. We can clearly see that the waves gain amplitude during propagation in Figure 7(a). Similar comparison between the measured and calculated waveforms is carried out for the $c$-mode envelope pulses in Figure 8. The upper and lower waveforms were monitored for the carrier frequency of $2.5 \mathrm{MHz}$ at the 5 th and 10th cells, respectively. In contrast to the $\pi$-mode waves, the $c$-mode ones lose amplitude during propagation. In both cases, the calculated waveforms well simulate the measured ones, including the amplitude gain and dispersive waveform distortions. By varying the carrier frequency of the input envelope pulses and voltage fractions, we obtain the frequency dependence of the per-unit-cell gain in Figure 9. Circles and squares represent the measured and calculated results for the $\pi$-mode inputs, respectively. The similarity between the measured and calculated results is established, so that we conclude that the numerical evaluations predict sufficiently the quantitative performance of CRLH TWFETs.

To discriminate the $c$ - and $\pi$-modes clearly, we carried out calculations of a large-size line. We set the total cell number to 500 and an envelope pulse with the carrier frequency of $2.3 \mathrm{MHz}$ was input to the 1 st cell of the gate

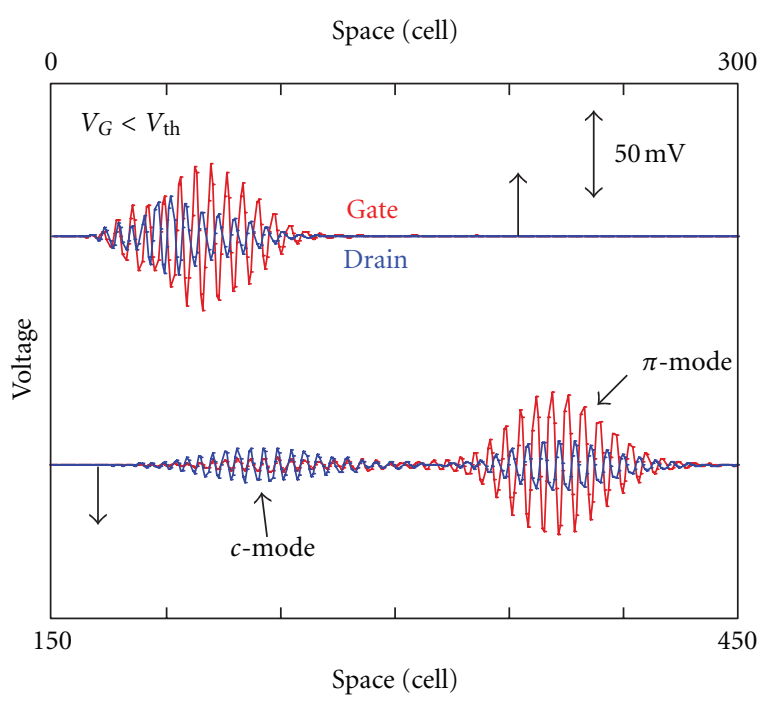

(a)

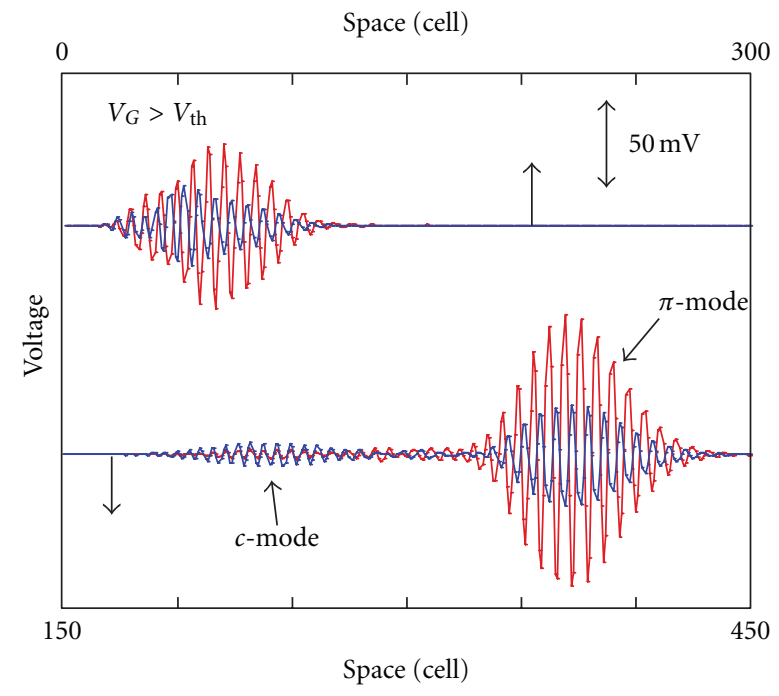

(b)

FIGURE 10: Numerically obtained envelope pulses in large CRLH TWFET. The total cell number is set to 500 without altering line parameters that were used to obtain Figures 6(b) and 7(b).

line. The pulse was split into the $c$ - and $\pi$-mode components, which were well separated in space after the long-length propagation. Figures 10 (a) and 10 (b) show the calculated results for $V_{G}<V_{\text {th }}$ and $V_{G}>V_{\text {th }}$, respectively. Upper and lower waveforms correspond to the pulses before and after the long-length propagation, respectively. The $\pi$-mode components preceded the $c$-mode ones and was amplified when $V_{G}>V_{\text {th }}$. On the other hand, it is clearly seen that the $c$-mode components was more attenuated for $V_{G}>V_{\text {th }}$ than for $V_{G}<V_{\text {th }}$.

\section{Numerical Performance of CRLH TWFETs}

We evaluate numerically the performance of a CRLH TWFET including series inductors. We employed the same reactance values as those used to obtain Figures 5 and 6 . 


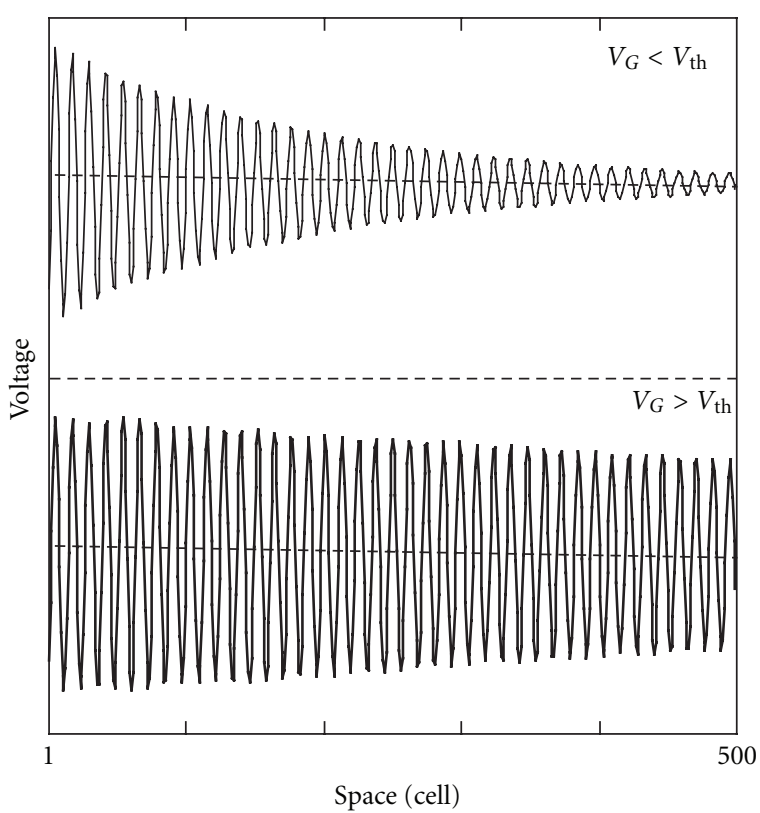

(a)

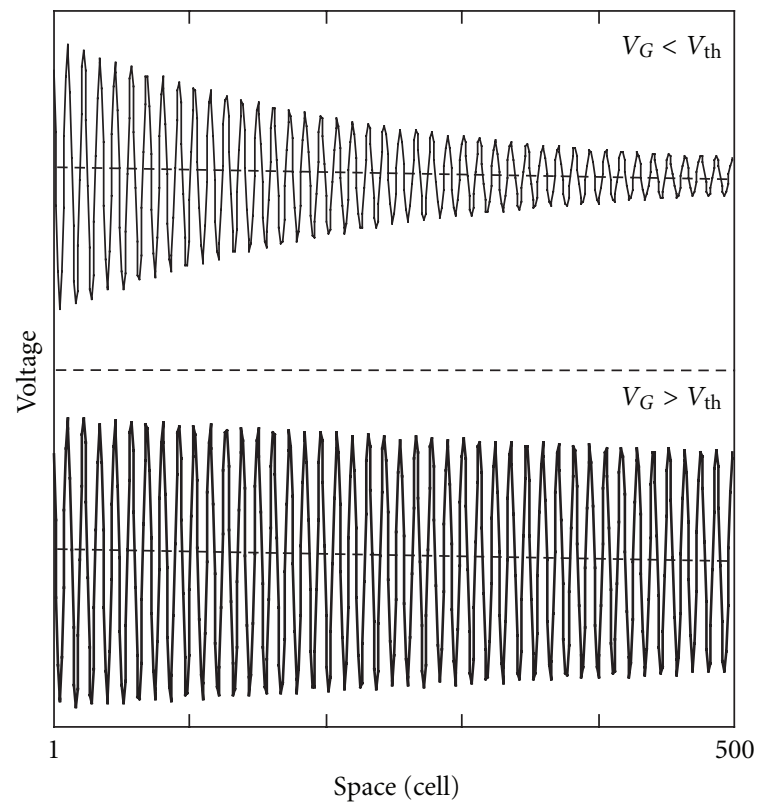

(b)

FIgURE 11: Steady-state waveforms in a CRLH TWFET. (a) RH and (b) LH waves. The thin and thick waveforms correspond to $V_{G}<$ $V_{\text {th }}$ and $V_{G}>V_{\text {th }}$, respectively.

The parasitic resistance and the gate bias voltage were tuned to clearly see the contributions of FET gain. It is expected that the line can amplify both the LH and RH waves, which are supported by the $\pi$ - and $c$-modes, respectively. The numerical model we developed is equally applied not only to the $\mathrm{MHz}$ circuits but also to the microwave circuits, when the print-circuit-board techniques are effectively utilized; therefore, the results obtained by our model can be considered to be sufficiently reliable for these parameter values. Responses to the sinusoidal inputs were evaluated, considering practical situations where CRLH TWFETs would be applied. The thick and thin waveforms in Figure 11(a) correspond to the steadystate LH sinusoidal waves for $V_{G}>V_{\text {th }}$ and for $V_{G}<V_{\text {th }}$, respectively. The frequency was set to $3.2 \mathrm{GHz}$, where the $\pi$-mode was supposed to be uniquely amplified. We thus designed terminal resistances to be matched with the $\pi$ mode characteristic impedances. Wave attenuation was well compensated by the FET contribution.

Finally, the properties of steady-state RH sinusoidal waves are shown in Figure 11(b). The frequency was set to $5.8 \mathrm{GHz}$. According to Figure 6 , the $c$-mode is uniquely amplified, so that we set the terminations to be matched with the c-mode impedance. Compensation of attenuation was also established for the $\mathrm{RH}$ waves.

In both cases, it was confirmed that development of standing waves was well suppressed by the matched terminations.

\section{Conclusions}

We characterize waves in CRLH TWFETs. We found that one of the possible two propagation modes is uniquely amplified for wide range of frequency. By the terminations with the characteristic impedance corresponding to the amplifying mode, the multiple reflections of waves can be effectively suppressed. These design criteria may be practically useful to produce large-scale platform to support both the LH and $\mathrm{RH}$ waves.

\section{References}

[1] C. Caloz and T. Itoh, Electromagnetic Metamaterials: Transmission Line Theory and Microwave Applications, WileyInterscience, New York, NY, USA, 2006.

[2] F. P. Casares-Miranda, C. Camacho-Peñalosa, and C. Caloz, "High-gain active composite right/left-handed leaky-wave antenna," IEEE Transactions on Antennas and Propagation, vol. 54, no. 8, pp. 2292-2300, 2006.

[3] L.-M. Si, T. Jiang, K. Chang, X. Lv, L. Ran, and H. Xin, "Active microwave metamaterials incorporating ideal gain devices," Materials, vol. 4, no. 1, pp. 73-83, 2010.

[4] K. Maezawa, K. Kasahara, and M. Mori, "A traveling wave amplifier based on composite right/left handed (CRLH) transmission lines periodically loaded with resonant tunneling diode pairs," in Proceedings of the 22nd International Conference on Indium Phosphide and Related Materials (IPRM '10), pp. 362365, June 2010.

[5] S. Nakagawa and K. Narahara, "Characterization of left-handed traveling-wave transistors," IEICE Transactions on Electronics, vol. E92-C, no. 11, pp. 1396-1400, 2009.

[6] K. C. Gupta, R. Garg, and I. J. Bahl, Microstrip Lines and Slotlines, Artech, London, UK, 1979.

[7] S. Nakagawa and K. Narahara, "Compensation of wave attenuation in left- handed traveling-wave field-effect transistors," Progress in Electromagnetics Research Letters, vol. 28, pp. 195205, 2012. 

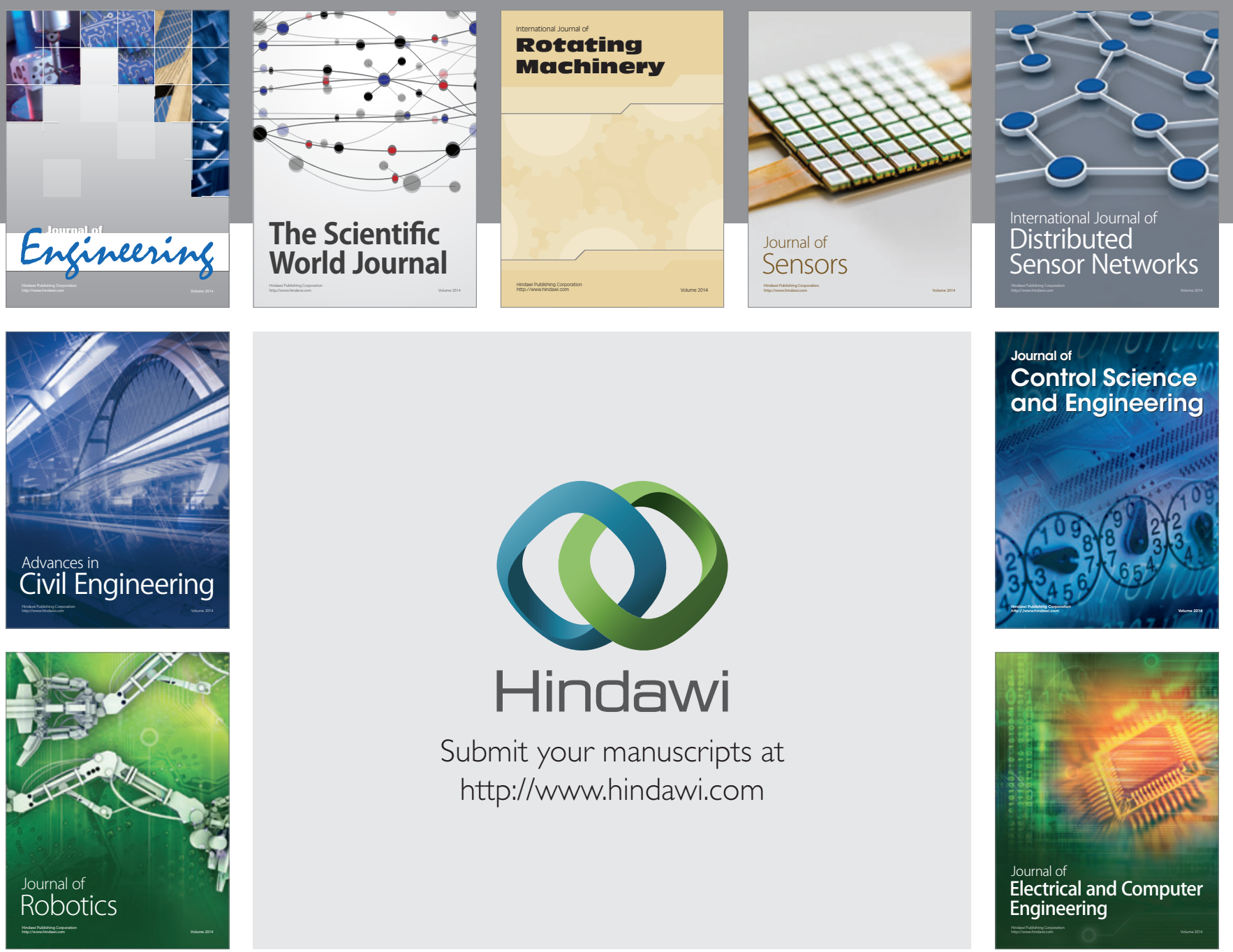

Submit your manuscripts at

http://www.hindawi.com
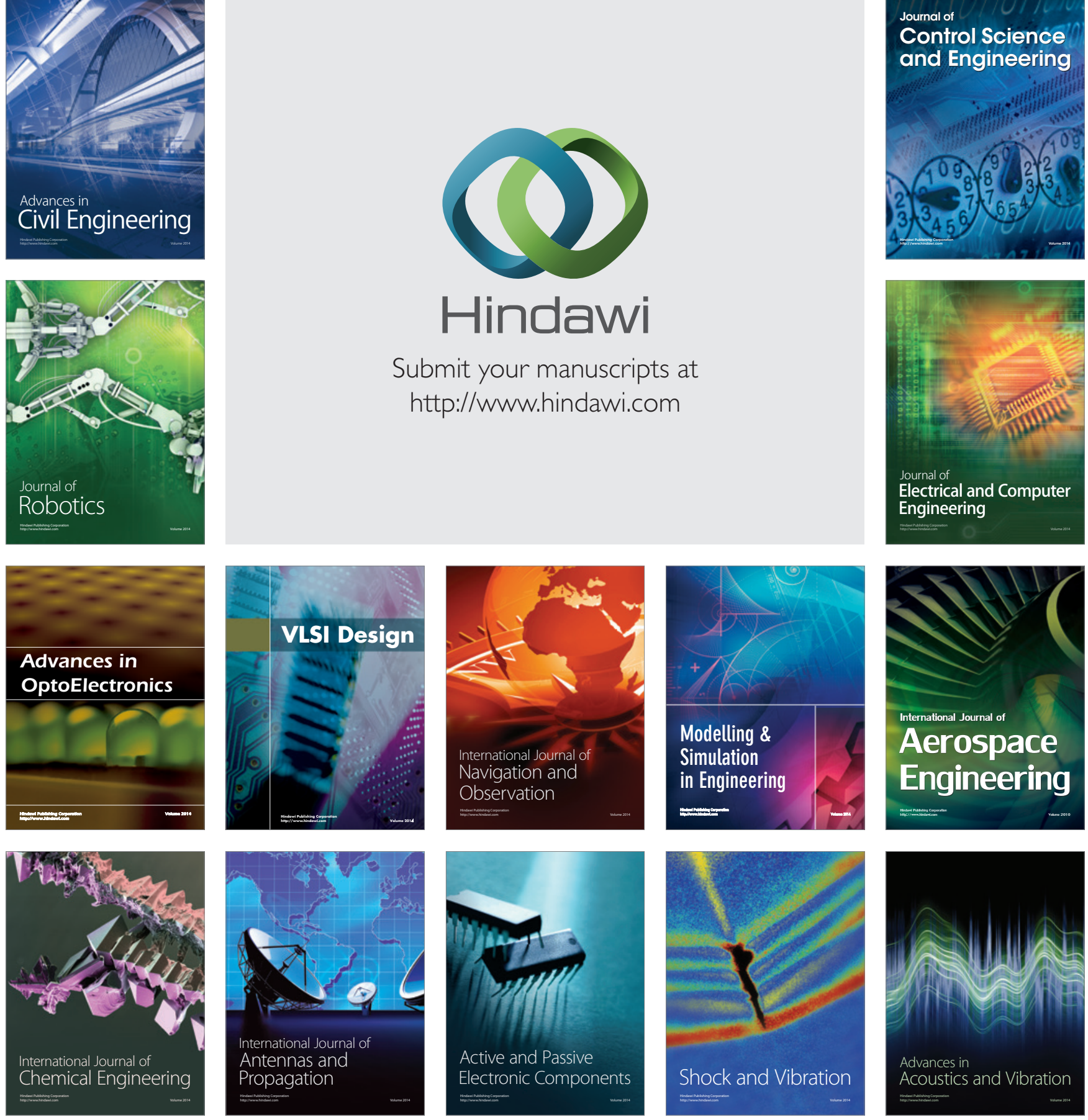